\title{
THE ESTIMATION OF SOME DETERMINANTS OF TOEPLITZ-TYPE
}

\author{
H. O. POLLAK AND L. A. SHEPP
}

1. Given a nonconstant real continuous function $\rho(t),-1 \leqq t \leqq 1$, we form, for $n=1,2, \cdots$, the matrix $\rho_{n}$ whose $(i, j)$ th element is $\rho((i-j) / n), i=0, \cdots, n, j=0, \cdots, n$. The purpose of this note is to estimate the determinants $\left(\left|\rho_{n}\right|\right)$ of $\rho_{n}$ for large $n$ for some specific functions $\rho$. We prove the following theorem.

TheOREM. If $\rho(t)=\rho(-t)$ has a uniformly bounded second derivative, $0<t<1$, and satisfies the following additional assumptions:

(i) $\rho^{\prime \prime}(t) \geqq 0,0<t<1$,

(ii) $\rho^{\prime}(t) \leqq 0,0<t<1$,

(iii) $\rho(1)>0$, then

$$
\left.\left|\rho_{n}\right| \sim \sim 2\left|\rho^{\prime}\left(0^{+}\right)\right| / n\right\}^{n}
$$

Here, sequences $a_{n}$ and $b_{n}, n=1,2, \cdots$ of positive numbers are said to be of the same order of growth, $a_{n} \sim \sim b_{n}$, if $a_{n}=O\left(b_{n}\right)$ and $b_{n}=O\left(a_{n}\right)$. The right-hand derivative of $\rho$ at zero, $\rho^{\prime}\left(0^{+}\right)$, exists and is strictly negative as a consequence of the hypothesis. We remark that the theorem becomes false if one drops the assumption of a uniformly bounded second derivative and replaces it with the assumption that $\rho$ be convex for $0 \leqq t \leqq 1$, and $\left|\rho^{\prime}\left(0^{+}\right)\right|<\infty$. It would seem that the strict inequality in (iii) could be relaxed, but we have not succeeded in doing this.

The problem of determining the order of growth of $\left|\rho_{n}\right|$ has arisen in some work of one of the authors on the discrimination of Gaussian processes [3]. The application of the present results to that study is quite direct and will appear elsewhere.

2. The method of proof is to use elementary row and column operations in order to bring the matrix into nearly unit-matrix form. In the remainder of the proof we use some lemmas of Price [2] and Ostrowski [1].

We subtract column $j-1$ from column $j$ in $\rho_{n}, j=n, n-1, \cdots, 1$ and thereafter subtract row $i-1$ from row $i, i=n, n-1, \cdots, 1$ in this order. We obtain a symmetric matrix $a=a_{n}$ whose general element $a_{i j}$ is

Presented to the Society, January 27, 1965; received by the editors August 31, 1964. 


$$
\begin{aligned}
& a_{i j}=2 \rho\left(\frac{i-j}{n}\right)-\rho\left(\frac{i-j-1}{n}\right)-\rho\left(\frac{i-j+1}{n}\right) \\
& i=1, \cdots, n, j=1, \cdots, n, \\
& a_{00}=\rho(0), \quad a_{k 0}=a_{0 k}=\rho\left(\frac{k}{n}\right)-\rho\left(\frac{k-1}{n}\right) \\
& k=1, \cdots, n .
\end{aligned}
$$

We denote by $a^{\prime}=a_{n}^{\prime}$ the $n \times n$ matrix obtained from $a$ by deleting the row $i=0$ and column $j=0$ from $a$. We next divide each of the rows of the deleted matrix, $a^{\prime}$, by the common diagonal entry $2(\rho(0)-\rho(1 / n))$, obtaining the matrix $b=b_{n}$ whose general element $b_{i j}$ is

(2.3) $b_{i j}=a_{i j} /(2 \rho(0)-2 \rho(1 / n)), \quad i=1, \cdots, n, \quad j=1, \cdots, n$.

From standard theorems on determinantal manipulations we have

$$
\begin{aligned}
& \left|\rho_{n}\right|=\left|a_{n}\right| \\
& \left|a_{n}^{\prime}\right|=\left(2 \rho(0)-2 \rho\left(\frac{1}{n}\right)\right)^{n}\left|b_{n}\right| .
\end{aligned}
$$

From (i) and (ii) by convexity and monotonicity of $\rho$ we see that $a_{i j} \leqq 0$ for $i \neq j$. It then follows that for $i=1,2, \cdots, n$,

$$
\begin{aligned}
\sum_{j \neq i}\left|a_{i j}\right|= & 2(\rho(0)-\rho(1 / n)) \\
& -\left(\rho\left(\frac{n-i}{n}\right)-\rho\left(\frac{n-i+1}{n}\right)\right) \leqq a_{i i}
\end{aligned}
$$

and

$$
\sum_{j \neq 0}\left|a_{0 j}\right|=\rho(0)-\rho(1) \leqq \rho(0)=a_{00} .
$$

The hypothesis of the following lemma of Price is satisfied.

LEMMA 1. If $a=a_{i j}, i=0, \cdots, n, j=0, \cdots, n$, is any real matrix for which $\sum_{j \neq i}\left|a_{i j}\right| \leqq a_{i i}, i=0, \cdots, n$, then

$$
\left(a_{00}-\sum_{j=1}^{n}\left|a_{0 j}\right|\right)\left|a^{\prime}\right| \leqq|a| \leqq\left(a_{00}+\sum_{j=1}^{n}\left|a_{0 j}\right|\right)\left|a^{\prime}\right|
$$

where $a^{\prime}$ is the matrix derived from $a$ by deleting the row $i=0$ and the column $j=0$. 
The lemma is only implicitly contained in [2] but the reader will have no difficulty in extracting it (see especially formula (13) on p. 501).

Applying (2.7) to $a_{n}$ we obtain

$$
\rho(1)\left|a_{n}^{\prime}\right| \leqq\left|a_{n}\right| \leqq(2 \rho(0)-\rho(1))\left|a_{n}^{\prime}\right| .
$$

To estimate the determinant $|b|$ in (2.3) we use the following special case of a theorem of Ostrowski [1].

Lemma 2. If $b=b_{i j}, i=1, \cdots, n, j=1, \cdots, n$, is any real symmetric matrix with $b_{i i}=1$ and

$$
\sum_{j \neq i}\left|b_{i j}\right| \leqq s \leqq 1, \quad i=1, \cdots, n
$$

then

$$
e^{8^{\sigma^{2 / s}}}(1-s)^{\sigma^{2} / 8^{2}} \leqq|b| \leqq 1
$$

where

$$
\sigma^{2}=\sum_{i \neq j} b_{i j}^{2}
$$

The left-hand inequality in (2.9) is proved in [1] even without assuming that $b$ is symmetric. It is necessary to assume symmetry for the right-hand inequality which seems to be new. Furthermore, in this case we give a simpler proof of the left-hand inequality. It is well-known and easy to see that all the eigenvalues of $b$ are non-negative. With $\sum_{\lambda}$ denoting summation over all eigenvalues of $b$, we have

$$
\operatorname{det} b=\exp \sum_{\lambda} \log \lambda \leqq \exp \sum_{\lambda}(\lambda-1)=1
$$

since $\sum_{\lambda} \lambda=$ trace $b=n$. The left-hand inequality is proved similarly, using the fact that for $s<1, \lambda \geqq 1-s$ and $\log \lambda \geqq \lambda-1-C(\lambda-1)^{2}$ for an appropriate value of $C$.

Using (2.5) to check that the hypothesis of Lemma 2 holds we may write (2.9) with $b=b_{n}$. Here $\sigma^{2}=\sigma_{n}^{2}$ is given by (2.10) and

$$
\begin{aligned}
s=s_{n}=\max _{i}\{1- & {\left[\rho\left(\frac{n-i}{n}\right)-\rho\left(\frac{n-i+1}{n}\right)+\rho\left(\frac{i}{n}\right)\right.} \\
& \left.\left.-\rho\left(\frac{i-1}{n}\right)\right] /\left(2 \rho(0)-2 \rho\left(\frac{1}{n}\right)\right)\right\} .
\end{aligned}
$$

Using Taylor's theorem we obtain 


$$
\rho(0)-\rho\left(\frac{1}{n}\right)=-\rho^{\prime}\left(0^{+}\right) / n+O\left(1 / n^{2}\right)
$$

and this yields

$$
\left(2 \rho(0)-2 \rho\left(\frac{1}{n}\right)\right)^{n} \sim\left(2\left|\rho^{\prime}\left(0^{+}\right)\right| / n\right)^{n} .
$$

Using (2.4), (2.8) then (2.5), (2.13) and (2.9) it is clear that we need only show that as $n \rightarrow \infty$ the extreme left-hand side of (2.9) remains bounded away from zero.

By a mean value theorem we have $a_{i j}=-\rho^{\prime \prime}\left(\theta_{i j}\right) / n^{2}$ where

$$
(i-j-1) / n<\theta_{i j}<(i-j+1) / n, \quad i \neq j .
$$

With $M=\sup \rho^{\prime \prime}$ we have $\left|b_{i j}\right| \leqq M / n^{2}(2 \rho(0)-2 \rho(1 / n))$ and it follows that

$$
\lim \sup \sigma_{n}^{2} \leqq M^{2} / 4\left|\rho^{\prime}\left(0^{+}\right)\right|^{2}
$$

In order to complete the proof of the theorem we need only bound $s=s_{n}$ away from unity (N.B. for $s$ near zero the extreme left-hand side of $(2.9)$ is not vanishingly small). If $\rho^{\prime}\left(1^{-}\right)<0$ we may proceed as follows. The mean value theorem and the fact that $\left|\rho^{\prime}(t)\right| \geqq\left|\rho^{\prime}\left(1^{-}\right)\right|$ gives easily

$$
\lim \sup s_{n} \leqq 1-\frac{2\left|\rho^{\prime}\left(1^{-}\right)\right|}{2\left|\rho^{\prime}\left(0^{+}\right)\right|}<1 .
$$

If now $\rho^{\prime}\left(1^{-}\right)=0$ the argument is more difficult. One must consider an appropriate power, $r$, of the matrix $b$ and apply Lemma 2 to $b^{r}$. For a sufficiently large fixed $r$ one shows that there is a $\delta>0$, independent of $n$ sufficiently large, such that for all $i$,

$$
\sum_{i \neq j}\left|b_{i j}^{(r)}\right| \leqq s<1
$$

The details are straightforward but cumbersome and are omitted.

\section{REFERENCES}

1. A. Ostrowski, Sur la détermination des bornes inférieures pour une classe des déterminants, Bull. Sci. Math. (2) 61 (1937), 19-32.

2. G. B. Price, Bounds for determinants with dominant principal diagonal, Proc. Amer. Math. Soc. 2 (1951), 497-502.

3. L. A. Shepp, The singularity of Gaussian measures in function space, Proc. Nat. Acad. Sci. 52 (1964) 430-433.

Bell Telephone laboratories, Inc., Murray Hill, New Jersey 